\title{
The lithium isotope ratio and beryllium in halo stars
}

\author{
Megan C. Novicki ${ }^{1}$ and Ann M. Boesgaard ${ }^{1}$ \\ ${ }^{1}$ Institute for Astronomy, University of Hawaii, Honolulu, HI, 96822, USA \\ email: mnovicki,boes@ifa.hawaii.edu
}

\begin{abstract}
The rare light elements - Li, Be, and B - have a unique and highly coupled history in the Universe. A coordinated analysis of their abundances in very low metallicity stars can help us understand the inner workings of stars and can constrain models of Galactic chemical evolution. We measure the Be abundances of nine stars and the $\mathrm{Li}$ isotopic ratio of ten stars. We find three stars with interesting Be abundances and three stars with detectable ${ }^{6} \mathrm{Li}$.
\end{abstract}

Keywords. Stars: abundances, blue stragglers, stars: Population II, subdwarfs

\section{Introduction}

As part of a coordinated analysis of the rare light elements in very low metallicity halo stars, we have observed a number of stars in order to find their Be abundances and $\mathrm{Li}$ isotopic ratios (Novicki 2005). The purpose of measuring ${ }^{6} \mathrm{Li}$ is twofold; we wish to constrain the amount of depletion in halo stars on the Spite plateau, and we wish to compare the $\mathrm{Be}$ and ${ }^{6} \mathrm{Li}$ abundances in order to get a better understanding of the different formation mechanisms of those two isotopes.

In addition, the abundance of Be in halo stars with respect to the $\mathrm{Li}$ abundance and the metallicity can provide insights into the inner workings of these stars. As Be survives to greater depths of the stellar interior, the abundance of Be relative to the total $\mathrm{Li}$ abundance in a star can provide clues to the specific depletion mechanism at work within the star.

\section{The Li Isotopic Ratio}

We measured the Li isotopic ratio in ten halo stars using the high-dispersion spectrograph (HDS) (Noguchi et al. (2002)) on the 8.2m Subaru Telescope on Mauna Kea. We chose a slit width of $0.3^{\prime \prime}$ to obtain a spectral resolution of approximately 110,000 , and we integrated on each source until we obtained a signal-to-noise ratio $(\mathrm{S} / \mathrm{N})$ of at least 250 . This high $\mathrm{S} / \mathrm{N}$ and resolution are required to detect the ${ }^{6} \mathrm{Li}$ component of the $6708 \AA \mathrm{Li} \mathrm{I}$ line. Of the ten stars in our sample, we detect ${ }^{6} \mathrm{Li}$ in three of them: HD 84937, G 126-52, and $\mathrm{BD}+2$ 4651. The latter two are new detections. We show G 126-52 in Figure 1. We conclude from these detections that $\alpha+\alpha$ reactions dominated over cosmic ray spallation reactions to form ${ }^{6} \mathrm{Li}$ at these levels. Also, we believe that the $\mathrm{Li}$ abundances of these stars cannot have been dramatically depleted over their lifetimes.

\section{Be in Halo Stars}

We measured the Be abundances of nine halo stars with a range of metallicity again using HDS. Using a $0.7 \times 4.4^{\prime \prime}$ slit, we achieved a spectral resolution of $\sim 50,000$. Six of the stars we observed have Be abundances which are consistent with previous measurements 

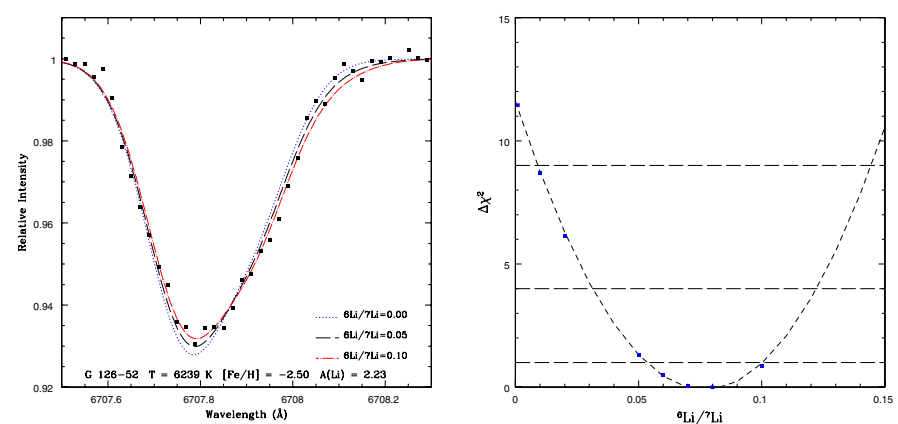

Figure 1. G 126-52 One of our new ${ }^{6} \mathrm{Li}$ detections $\left({ }^{6} \mathrm{Li} /{ }^{7} \mathrm{Li}=0.07 \pm 0.02\right)$ : the $6707 \AA$ doublet on the left, and the $\Delta \chi^{2}$ as a function of isotopic ratio on the right.

from the literature (see Boesgaard, 2004). The other three stars have interesting Be abundances with respect to other stars and/or their Li abundances.

HD 132475 We find the Be abundance for this star is $\mathrm{A}(\mathrm{Be})=0.71$, which is well above the abundance expected for a star with $[\mathrm{Fe} / \mathrm{H}]=-1.5$. This star also has a $\mathrm{Li}$ abundance well above the Spite plateau value (Novicki 2005). Another star, BD +23 3912, also has an elevated Li abundance, but has a "normal" Be abundance and similar effective temperature, $[\mathrm{Fe} / \mathrm{H}]$, and surface gravity.

G 64-37 This star has been the subject of much discussion in the literature due to the fact that it has very low metallicity, -3.2 . It is similar in its physical properties to another star, G 64-12, but G 64-37 has an A(Li) which is lower by 0.3dex (Novicki 2005). We measured $\mathrm{A}(\mathrm{Be})<-1.3$ for $\mathrm{G} 64-37$, which is less than the $\mathrm{A}(\mathrm{Be})=-1.15$ found by Primas et al. (2000) for G 64-12.

G 186-26 There are nine stars known to be "ultra-Li-deficient", i.e. have A(Li) significantly below the plateau value. G $186-26$ is one of these nine, and has a very low metallicity: $[\mathrm{Fe} / \mathrm{H}]=-2.71$. We find that this star is also Be-deficient, and we place an upper limit on its Be abundance to be $\mathrm{A}(\mathrm{Be})=-2$. Of the two theories of Be depletion in stars, we favor the blue-straggler analog theory over rotationally induced mixing. For a further discussion on this topic, see Boesgaard \& Novicki (2005).

\section{Conclusions}

Using the HDS on the Subaru Telescope we observed the Li isotopic ratio in 10 metalpoor stars and found evidence for ${ }^{6} \mathrm{Li}$ in three of them. These detections indicate that the $\alpha+\alpha$ process was probably dominant in the early Galactic halo.

We also observed nine stars for their Be abundances, six of which are consistent with increasing Be with respect to $[\mathrm{Fe} / \mathrm{H}]$. HD 132475 has a Be abundance which is above this relation and also has a $\mathrm{Li}$ abundance 0.2 dex above the plateau in Li. The ultra-Lideficient star G $186-26$ is also ultra Be-deficient, so it is perhaps connected to the blue straggler phenomenon.

\section{Acknowledgements}

This research was supported by NSF under grant number AST-0097945.

\section{References}

Boesgaard, A.M. 2004, in Origin and Evolution of the Elements, 119-+z 
Boesgaard, A.M. \& Novicki, M.C. 2005, submitted

Noguchi, K. et al. 2002, PASJ, 54, 855

Novicki, M.C. 2005, dissertation, University of Hawaii

Primas, F., Asplund, M., Nissen, P.E., \& Hill, V. 2000, A\& A, 364, L42 


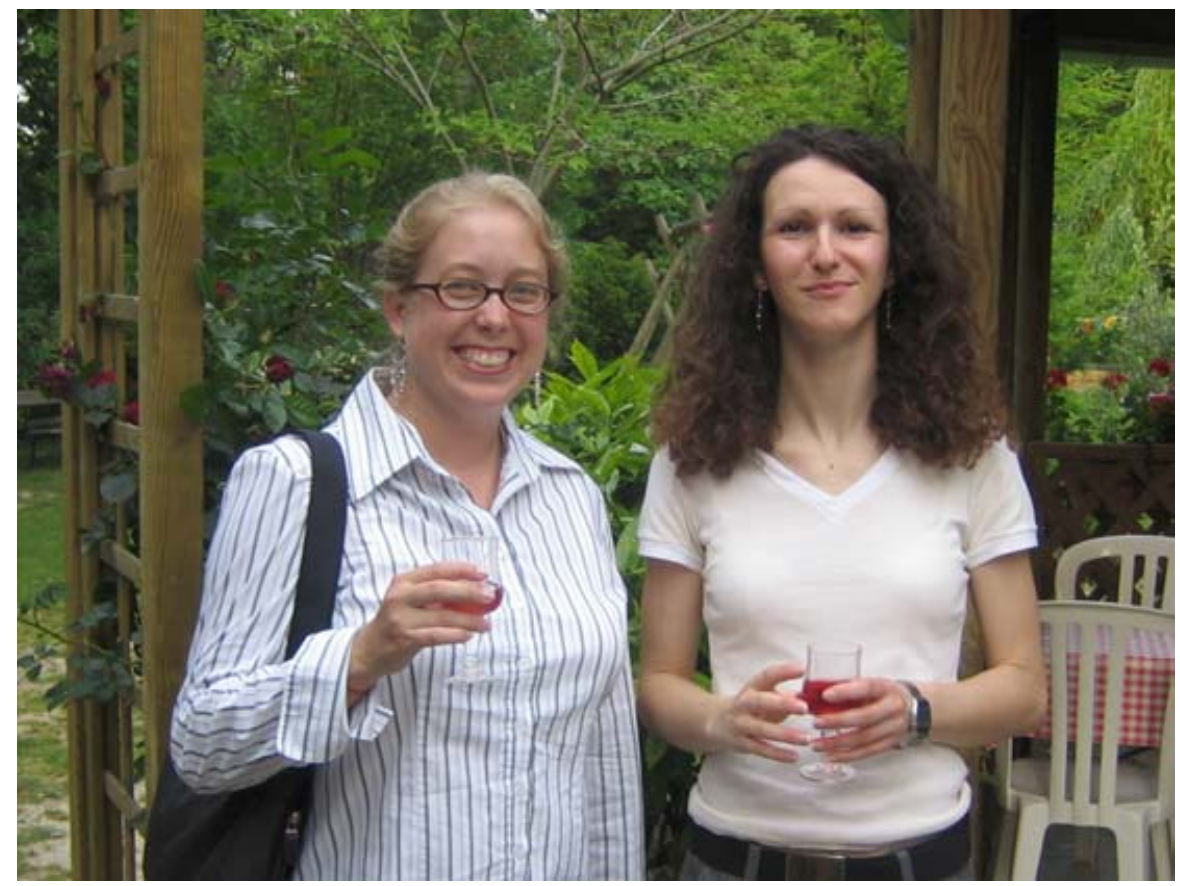

Megan Novicki and Sara Bisterzo, discussing at the aperitif before the conference dinner.

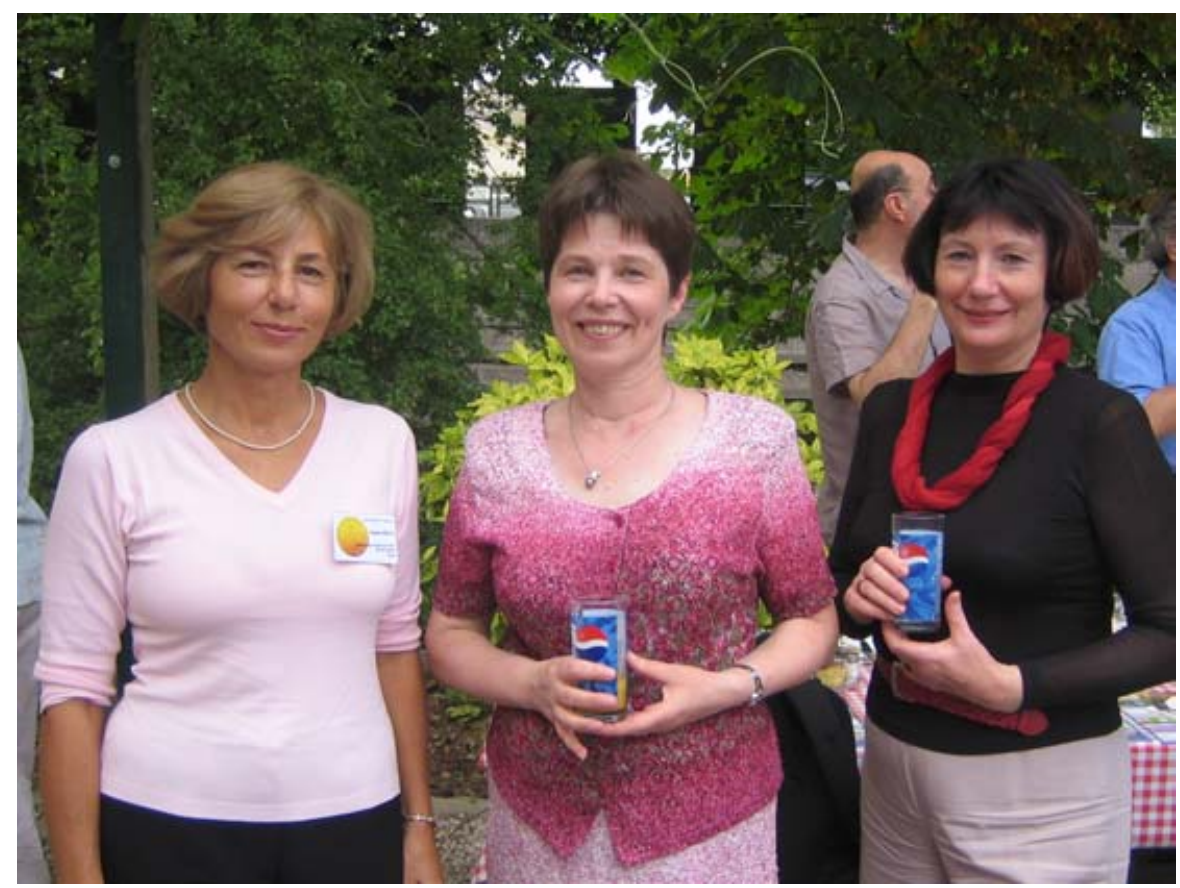

Natalia Drake, Lyudmila Mashonkina and Tamara Mishenina at the conference dinner. 